\title{
An Improved Spectrum Sensing for Cognitive PLC Systems
}

\author{
Alam S. Menezes, Yan F. Coutinho, and Moisés V. Ribeiro
}

\begin{abstract}
This paper introduces an improved spectrum sensing technique that exploits the existing information in the signals received from both electric power grids and wireless media to allow power line communication systems to monitor the spectrum occupancy/holes in the frequency band between 1.7 and $100 \mathrm{MHz}$. The applied procedure for implementing the proposed technique is organized in time-frequency mapping, selection and extraction of quanta, features extraction and selection, and detection. The attained results based on a measured data set show that the performance of techniques based on neural networks is far superior when compared to the techniques based on Bayes.
\end{abstract}

Keywords - Power line communication, smart grids, spectrum sensing, digital communication.

\section{INTRODUCTION}

Power Line Communication (PLC) is being pointed out as one of the technologies to meet the data communication demands related to Smart Grids (SG), Smart Cities (SC), Internet of Things (IoT), and Industry 4.0 [1]. Also, the capillarity of the electric power systems allows the integration of several sensor devices into the utility's supervision and control platform through PLC networks [2]. However, it is well-established that electric power systems have been designed for power transmission and distribution at very low frequencies (e.g., $60 \mathrm{~Hz}$ in Brazil). For signals with spectral content at high frequencies, electric power circuits considerably attenuate the signal propagation in a dynamic manner. In addition, electromagnetic interference and compatibility with other telecommunications equipment operating in the same frequency range as the PLC device - both physically connected to electric power circuits - constitute severe concerns for the use of electric power systems for data communication purposes [3]. The hostile characteristics of electric power systems for transmitting carrying-signals information and the existing regulatory constraints motivate flexible spectrum access, which is based on the cognitive radio (CR) concept [4], for dealing with the unique characteristics of PLC systems [5].

In this sense, several researches investigated the CR techniques in PLC systems. The power line was used as a sensor in [6] to detect spectrum holes and to avoid possible interference

This study was supported in part by Coordenação de Aperfeiçoamento de Pessoal de Nível Superior (CAPES) - finance code 001, in part by Conselho Nacional de Desenvolvimento Científico e Tecnológico (CNPq), in part by Instituto Nacional de Energia Elétrica (INERGE), and in part by Fundação de Amparo à Pesquisa do Estado de Minas Gerais (FAPEMIG).

Alam S. Menezes was with the Department of Electrical Engineering, Federal University of Juiz de Fora, Juiz de Fora, MG 36036 330, Brazil, and is with the Engineering Department of Petrobras Transporte, Rio de Janeiro, RJ, Brazil (e-mail: alamsm@petrobras.com.br).

Yan F. Coutinho and Moisés V. Ribeiro are with the Department of Electrical Engineering, Federal University of Juiz de Fora, Juiz de Fora, MG 36036 330, Brazil (e-mail: \{yan.coutinho,mribeiro\}@engenharia.ufjf.br). in the radio communication systems. Based on measured data, [7] applied the time-frequency analysis of the monitored signal in the power line, as well as a detection threshold based on the average power of the noise in the monitored frequency bandwidth to detect the occupied frequency bands. Also, in [8], a spectral monitoring technique using an adaptive threshold for the detection of holes in the spectrum was formulated. In general, spectral monitoring in the PLC system uses only signal extracted from a power line. However, [9] analyzed the use of signals extracted from power line and air, which is accomplished with the use of an antenna, for spectrum sensing purposes [10].

Thus, aiming to improve the spectrum sensing for cognitive PLC systems, this paper introduces an improved spectrum sensing analysis. For this purpose, sensors are used to acquire both wireless and power line signals using, respectively, an omnidirectional antenna and a PLC coupler, which is connected to the low-voltage electric power circuit. In this sense, we propose the time-frequency analysis of the signal extracted from both power line and antenna, and the spectrograms formed from such analysis are subdivided into small portions called, in the present work, quanta. Using feature extraction and selection techniques, as well as detection techniques based on Bayes and multilayer perceptron (MLP) neural networks, spectrum holes are detected in each quantum. Finally, we present a comparative evaluation of the proposed technique, based on the use of measured data from PLC coupler and antenna as sensors, covering the frequency band between 1.7 and $100 \mathrm{MHz}$. Numerical results show that the MLP-based configurations offer better performance than the Bayes-based ones. Also, the performance discrepancy is relatively high when comparing the quanta corresponding to the PLC coupler and the ones corresponding to the antenna in some technique configurations.

\section{PlC Spectrum Sensing Modeling}

Let the baseband frequency range $0 \leq f \leq B \mathrm{~Hz}$ be occupied by a PLC system. In order to map the sub-bands occupied by the primary user (PU) and to monitor the spectrum access dynamics, the cognitive power line (CPLC) system must be able to perform a time-frequency analysis of the monitored signal. To do so, we consider that the formulation of the spectral monitoring problem from both PLC coupler and antenna sensors is the same. Thus, without loss of generality, the signal at the input of only one sensor element can be expressed as

$$
y(t)=\sum_{k=1}^{K} s_{k}(t)+v(t),
$$


where $s_{k}(t)$ is the $k^{t h}$ signal from a PU, whereas $y(t)$ is a signal in the time domain corrupted by the additive noise $v(t)$ at the input of the sensor element (e.g., antenna or PLC coupler). The acquisition process of the $y(t)$ signal can be performed by different acquisition devices (transducer operating with an acquisition board and analog-to-digital converter) associated with a given sensor (antenna or PLC coupler). In this sense, the acquisition of the signal can be modeled by

$$
\begin{aligned}
\mathbf{y} & =\mathcal{T}_{m}\{y(t)\} \\
& =\mathcal{T}_{m}\left\{\sum_{k=1}^{K} s_{k}(t)\right\}+\mathcal{T}_{m}\{v(t)\} \\
& =\mathbf{s}+\mathbf{v},
\end{aligned}
$$

where $\mathcal{T}_{m} \in\left\{\mathcal{T}_{a}, \mathcal{T}_{c}\right\}$ denotes the used sensor, which encompasses all components from the transductor up to the digitizer, used for the acquisition of a signal from the air or the power line. Also, $\mathbf{y} \in \mathbb{R}^{N \times 1}, \mathbf{s} \in \mathbb{R}^{N \times 1}$, and $\mathbf{v} \in \mathbb{R}^{N \times 1}$ are vectors consisting of $N$ elements, which are $N$ consecutive samples obtained by digitization of the signals $y(t), s_{k}(t)$, and $v(t)$ (i.e., $y[n], s_{k}[n]$ and $v[n]$ ), respectively. The sampling rate is $2 B \mathrm{~Hz}$ since $|f| \leq B$.

In this work, (2) is rewritten in a matrix form as follows:

$$
\mathbf{M}_{\mathbf{y}}=\mathbf{M}_{\mathbf{s}}+\mathbf{M}_{\mathbf{v}}
$$

in which $\mathbf{M}_{\mathbf{s}}=\left[\mathbf{s}_{1} \mathbf{s}_{2} \cdots \mathbf{s}_{N-M}\right], \mathbf{M}_{\mathbf{v}}=\left[\mathbf{v}_{1} \mathbf{v}_{2} \cdots \mathbf{v}_{N-M}\right]$ and the column vectors of each matrix are defined as $\mathbf{y}_{n} \triangleq$ $[y[n] y[n+1] \cdots y[n+N-1]]^{T}, \mathbf{s}_{n} \triangleq[s[n] s[n+1] \cdots s[n+$ $N-1]]^{T}, \mathbf{v}_{n} \triangleq[v[n] v[n+1] \cdots v[n+N-1]]^{T}$ for $n=0,1, \cdots, N-M+1$. Note that column vectors represent segments that are stacked side by side to form the matrices $\mathbf{M}_{\mathbf{y}}, \mathbf{M}_{\mathbf{s}}$, and $\mathbf{M}_{\mathbf{v}}$ of order $N \times(N-M)$. Also, $n$ can be replaced by an expression to allow us to cover a long time interval while the choice of $N$ allow us to control the spectrum resolution, which is given by $2 B / N$.

\section{IMPROVED PLC SPECTRUM SENSING}

The block diagram in Fig. 1 schematically shows the proposed technique for spectrum monitoring in CPLC systems using antenna and PLC coupler sensors. It is organized in time-frequency mapping, selection and extraction of quanta, features extraction and selection, and detection. The detailed description of each one of the blocks are presented from Section III-A to Section III-E.

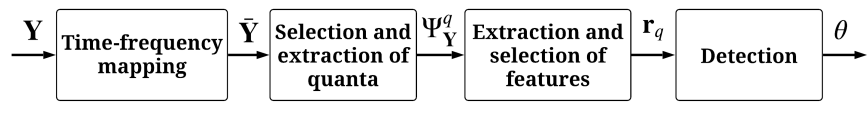

Fig. 1. Block diagram of the spectrum sensing technique.

\section{A. Time-frequency Mapping}

In order to perform the time-frequency analysis, the first step is to take the information from the monitored signal in the time domain to the time-frequency domain by means of timefrequency transform. Such procedure can be mathematically expressed as

$$
\begin{aligned}
\mathbf{M}_{\mathbf{Y}} & =\mathbf{A M}_{\mathbf{y}} \\
& =\mathbf{A M}_{\mathbf{s}}+\mathbf{A} \mathbf{M}_{\mathbf{v}} \\
& =\mathbf{M}_{\mathbf{S}}+\mathbf{M}_{\mathbf{V}},
\end{aligned}
$$

where $\mathbf{A}$ is a time-frequency transformation matrix, and $\mathbf{M}_{\mathbf{Y}} \in \mathbb{C}^{N \times(N-M)}, \mathbf{M}_{\mathbf{S}} \in \mathbb{C}^{N \times(N-M)}$ and $\mathbf{M}_{\mathbf{V}} \in$ $\mathbb{C}^{N \times(N-M)}$ are the monitored signals represented in the time-frequency domain. Note that each column of the matrix $M_{Y}$ represents a time window of the monitored signal, while each line represents a spectral component. The time-frequency transforms considered in the present work are the Discrete Fourier Transform (DFT), the Discrete Hartley Transform (DHT), the Modulated Complex Lapped Transform (MCLT), the Multitaper Method (MTM), and the Hartley Multitaper Method (HMTM).

In this sense, the stochastic energy spectral density (ESD) of the monitored signal represented in the time-frequency domain can be obtained by using

$$
\begin{aligned}
\mathbf{\Psi}_{\mathbf{Y}} & =\mathbf{M}_{\mathbf{Y}}^{*} \odot \mathbf{M}_{\mathbf{Y}} \\
& =\left(\mathbf{M}_{\mathbf{S}}+\mathbf{M}_{\mathbf{V}}\right)^{*} \odot\left(\mathbf{M}_{\mathbf{S}}+\mathbf{M}_{\mathbf{V}}\right) \\
& =\mathbf{\Psi}_{\mathbf{S}}+\mathbf{\Psi}_{\mathbf{C}}+\mathbf{\Psi}_{\mathbf{V}},
\end{aligned}
$$

where $\odot$ is the Hadamard operator and $(\cdot)^{*}$ denotes the complex conjugate operator. Note that $\mathbf{\Psi}_{\mathbf{S}}=\mathbf{M}_{\mathbf{S}}^{*} \odot \mathbf{M}_{\mathbf{S}}$ is the spectrogram of the monitored signal $\mathbf{M}_{\mathbf{S}}, \mathbf{\Psi}_{\mathbf{C}}=\mathbf{M}_{\mathbf{S}}^{*} \odot \mathbf{M}_{\mathbf{V}}+$ $\mathbf{M}_{\mathbf{V}}^{*} \odot \mathbf{M}_{\mathbf{S}}$ is the cross-spectrogram, and $\mathbf{\Psi}_{\mathbf{V}}=\mathbf{M}_{\mathbf{V}}^{*} \odot \mathbf{M}_{\mathbf{V}}$ is the spectrogram of the noise $\mathbf{M}_{\mathbf{V}}$.

\section{B. Tiles Extraction and Selection}

With the purpose of detecting PUs, we divide the spectrogram $\boldsymbol{\Psi}_{\mathbf{Y}}$ into small portions $\boldsymbol{\Psi}_{\mathbf{Y}}^{q} \in \mathbb{R}^{N_{F} \times N_{T}}$, forming a mosaic, where $\Psi_{\mathbf{Y}}^{q}$ is a two-dimensional geometric figure, as shown in Fig. 2 considering three different sizes of quanta. After the size of the quanta is chosen, the whole spectrogram is filled with several quanta. Note that $\boldsymbol{\Psi}_{\mathbf{Y}}^{r} \cap \boldsymbol{\Psi}_{\mathbf{Y}}^{s}=0, \forall r \neq s$ and $\boldsymbol{\Psi}_{\mathbf{Y}}=\bigcup_{q=0}^{Q} \boldsymbol{\Psi}_{\mathbf{Y}}^{q}$. In this mosaic, each portion $\boldsymbol{\Psi}_{\mathbf{Y}}^{q} \subset \boldsymbol{\Psi}_{\mathbf{Y}}$ is called the detection quantum. Each quantum is an $N_{F} \times N_{T}$ dimension matrix representing a small area of the spectrogram. The choice of quantum size is directly related to the spectrum usage time and the bandwidth of the monitored signal.

After dividing the spectrogram into a quanta mosaic, the problem of detecting the PUs in the $q^{t h}$ quantum may be formulated in two hypotheses

$$
\hat{\theta}=\left\{\begin{array}{l}
\mathcal{H}_{0} \rightarrow \boldsymbol{\Psi}_{\mathbf{Y}}^{q}=\mathbf{\Psi}_{\mathbf{V}}^{q} \\
\mathcal{H}_{1} \rightarrow \Psi_{\mathbf{Y}}^{q}=\Psi_{\mathbf{S}}^{q}+\Psi_{\mathbf{C}}^{q}+\Psi_{\mathbf{V}}^{q}
\end{array},\right.
$$

where $\mathcal{H}_{0}$ and $\mathcal{H}_{1}$ represent, respectively, the hypotheses of the absence or presence of PUs within the analyzed quantum. It can be seen that the quantum dimension $N_{F}$ represents the frequency resolution of the monitored band. Once the PU is identified, the range corresponding to the evaluated quantum is set as occupied. 


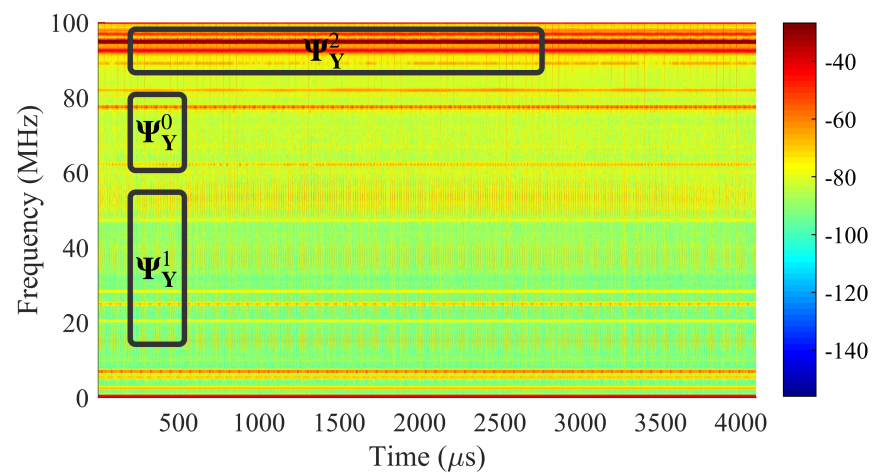

Fig. 2. Spectrogram with quanta.

\section{Features Extraction}

In order to extract a representative set of the monitored signal, it is possible, from the quantum of the monitored signal, to extract a vector given by

$$
\mathbf{r}_{q}=\Upsilon_{z}\left\{\boldsymbol{\Psi}_{\mathbf{Y}}^{q}\right\}
$$

where $\mathbf{r}_{q} \in \mathbb{R}^{K \times 1}$ and $K=N_{F} N_{T}$. The operator $\Upsilon_{z}\{\cdot\}$ represents a set of ways of forming the vector $\mathbf{r}_{q}$ with the elements of the matrix formed by the quantum $\mathbf{\Psi}_{\mathbf{Y}}^{q}$.

Considering that spectrum monitoring can be performed via antenna or PLC coupler, which, in general, implies the presence of Gaussian and non-Gaussian noise [11]-[13], second order as well as higher-order statistical features are used. Let us consider $\left\{r_{q}[k]\right\}_{k=0}^{N_{F} N_{T}-1}$, where $r_{q}[k]$ is the $k^{\text {th }}$ element of the vector $\mathbf{r}_{q}$, which is formed by the concatenation of a quantum $\Psi_{\mathbf{Y}}^{q}$. Therefore, the considered features are as follows:

- Cumulants: if $\mathbb{E}\left\{r_{q}[k]\right\}=0$, where $\mathbb{E}\{\cdot\}$ is the mean operator, the second, third, and fourth order cumulants, are expressed, respectively, as

$$
\begin{gathered}
c_{2, \mathbf{r}_{q}}[l]=\mathbb{E}\left\{r_{q}[k] r_{q}[k+l]\right\}, \\
c_{3, \mathbf{r}_{q}}[l]=\mathbb{E}\left\{r_{q}[k] r_{q}^{2}[k+l]\right\}, \\
c_{4, \mathbf{r}_{q}}[l]=\mathbb{E}\left\{r_{q}[k] r_{q}^{3}[k+l]\right\}-3 c_{2, \mathbf{r}_{q}}[l] c_{2, \mathbf{r}_{q}}[0],
\end{gathered}
$$

where $l$ refers to the $l^{\text {th }}$ delay in the evaluated data sequence. Their stochastic estimates are given by

$$
\begin{aligned}
& \tilde{c}_{2, \mathbf{r}_{q}}[l] \simeq \frac{2}{K} \sum_{k=0}^{K / 2-1} r_{q}[k] r_{q}[k+l], \\
& \tilde{c}_{3, \mathbf{r}_{q}}[l] \simeq \frac{2}{K} \sum_{k=0}^{K / 2-1} r_{q}[k] r_{q}^{2}[k+l], \\
& \tilde{c}_{4, \mathbf{r}_{q}}[l] \simeq \frac{2}{K} \sum_{k=0}^{K / 2-1} r_{q}[k] r_{q}^{3}[k+l] \\
& -\frac{12}{K} \sum_{k=0}^{K / 2-1} r_{q}[k] r_{q}[k+l] \sum_{k=0}^{K / 2-1} r_{q}^{2}[k],
\end{aligned}
$$

where $l=0,1, \cdots, K / 2-1$, and $\hat{c}_{2, \mathbf{r}_{q}}[l], \hat{c}_{3, \mathbf{r}_{q}}[l]$ and $\hat{c}_{2, \mathbf{r}_{q}}[l]$ are the stochastic approximations of the second, third and fourth order cumulants, respectively.

- Skewness: the skewness is given by

$$
\gamma_{3, \mathbf{r}_{q}}=\frac{\mathbb{E}\left\{\left(r_{q}[k]-\mu_{\mathbf{r}_{q}}\right)^{3}\right\}}{\left[\mathbb{E}\left\{\left(r_{q}[k]-\mu_{\mathbf{r}_{q}}\right)^{2}\right\}\right]^{3 / 2}},
$$

where $\mu_{\mathbf{r}_{q}}=\mathbb{E}\left\{r_{q}[k]\right\}$.

- Kurtosis: the Kurtosis is given by

$$
\kappa_{4, \mathbf{r}_{q}}=\frac{\mathbb{E}\left\{\left(r_{q}[k]-\mu_{\mathbf{r}_{q}}\right)^{4}\right\}}{\left(\mathbb{E}\left\{\left(r_{q}[k]-\mu_{\mathbf{r}_{q}}\right)^{2}\right\}\right)^{1 / 2}} .
$$

- Energy: the quantum energy is expressed as

$$
E_{\mathbf{r}_{q}}=\sum_{k=0}^{K-1}\left|r_{q}[k]\right|^{2}
$$

\section{Features Selection}

Features selection is the step of choosing the most relevant features for the detection process, contributing to reduce the dimensionality of the features vector, which is given by

$$
\mathbf{c}_{q \mid \mathcal{H}_{i}}=\left[\begin{array}{llllll}
\hat{c}_{2, \mathbf{r}_{q}} & \hat{c}_{3, \mathbf{r}_{q}} & \hat{c}_{4, \mathbf{r}_{q}} & \gamma_{3, \mathbf{r}_{q}} & \kappa_{4, \mathbf{r}_{q}} & E_{\mathbf{r}_{q}}
\end{array}\right]^{T},
$$

where $i=0,1$.

Assuming that each characteristic of a quantum is obtained from a sum of random variables, one can model $\mathbf{c}_{q}$ as a vector of independent random variables, with Gaussian distribution. In this way, for the two quantum states, the following probability density functions (PDFs) can be modeled:

$$
\begin{aligned}
& p_{\mathbf{c}_{q \mid \mathcal{H}_{0}}}=\mathcal{N}\left(\mathbb{E}\left\{\mathbf{c}_{q \mid \mathcal{H}_{0}}\right\}, \mathbf{K}_{\mathbf{c}_{q}}\right), \\
& p_{\mathbf{c}_{q \mid \mathcal{H}_{1}}}=\mathcal{N}\left(\mathbb{E}\left\{\mathbf{c}_{q \mid \mathcal{H}_{1}}\right\}, \mathbf{K}_{\mathbf{c}_{q}}\right),
\end{aligned}
$$

where $\mathbf{K}_{\mathbf{c}_{q}}$ is the autocovariance matrix of $\mathbf{c}_{q}$, whose diagonal is composed by the vector $\boldsymbol{\sigma}=\left[\sigma_{1}^{2} \cdots \sigma_{L_{f}}^{2}\right]$, in which $\sigma_{n}^{2}$ is the variance of the $n^{t h}$ feature, with $1 \leq n \leq L_{f}$, and $L_{f}$ is the total number of features. Based on this assumption, the Fisher Determinant Ratio (FDR) is used for the selection as follows:

$$
\boldsymbol{v}_{\mathbf{c}}=\left(\mathbb{E}\left\{\mathbf{c}_{q} \mid \mathcal{H}_{0}\right\}-\mathbb{E}\left\{\mathbf{c}_{q} \mid \mathcal{H}_{1}\right\}\right) \odot\left[\frac{1}{2 \sigma_{1}^{2}} \cdots \frac{1}{2 \sigma_{L_{f}}^{2}}\right],
$$

where $\boldsymbol{v}_{\mathbf{c}}=\left[\begin{array}{llll}v_{1} & v_{2} & \cdots & v_{L_{f}}\end{array}\right]^{T}$. The elements of the vector $\boldsymbol{v}_{\mathbf{c}}$ with the highest values indicate the features that will form the characteristic features vector $\mathbf{g}_{q}$ with reduced dimensionality for the $q^{\text {th }}$ quantum, as formulated below:

$$
\mathbf{g}_{q}=\mathcal{S}_{\max \boldsymbol{v}_{\mathbf{c}}}^{\zeta}\left\{\mathbf{c}_{q}\right\},
$$

in which $\mathcal{S}_{\max }^{\zeta} \boldsymbol{v}_{\mathrm{c}}\{\cdot\}$ represents the operation for selecting the $\zeta$ features associated with the highest values of the FDR vector $v_{\mathrm{c}}$.

In order to explore the diversity of the means of propagating signals through the air and the electric power cable, we propose to use the signals from the antenna sensors and PLC 
coupler. For this, we use the most relevant features from both the antenna and the PLC coupler combined as follows:

$$
\mathbf{z}_{q} \triangleq\left[\begin{array}{l}
\mathbf{g}_{q}^{a} \\
\mathbf{g}_{q}^{c}
\end{array}\right]
$$

where $\mathbf{z}_{q} \in \mathbb{R}^{L_{\mathbf{z}_{q}} \times 1}$ is a vector composed of selected features obtained from monitoring both the antenna $\left(\mathbf{g}_{q}^{a}\right)$ and the PLC coupler $\left(\mathbf{g}_{q}^{c}\right)$, concomitantly.

\section{E. Detection}

Quanta detection is the last stage of the cognitive cycle. For each set of features extracted from the quantum, represented by the vector $\mathbf{z}_{q}$ with reduced dimensionality, a classification technique is used to determine whether the analyzed quantum is occupied or not. The two states are represented by the hypotheses $\mathcal{H}_{1}$ and $\mathcal{H}_{0}$, respectively. For that, we use the detection technique based on the Bayes' theorem and the PDF of the data to obtain an analytical expression with the purpose of determining, with maximum likelihood, which of the hypotheses $\left(\mathcal{H}_{1}\right.$ or $\left.\mathcal{H}_{0}\right)$ the vector $\mathbf{z}_{q}$ is more related.

Thus, the rule for classifying a quantum in hypotheses $\mathcal{H}_{1}$ or $\mathcal{H}_{0}$ is expressed as

$$
p\left(\mathbf{z}_{q} \mid \mathcal{H}_{0}\right) P\left(\mathcal{H}_{0}\right)>p\left(\mathbf{z}_{q} \mid \mathcal{H}_{1}\right) P\left(\mathcal{H}_{1}\right), \mathbf{z}_{q} \rightarrow \mathcal{H}_{0},
$$

or

$$
p\left(\mathbf{z}_{q} \mid \mathcal{H}_{0}\right) P\left(\mathcal{H}_{0}\right) \leq p\left(\mathbf{z}_{q} \mid \mathcal{H}_{1}\right) P\left(\mathcal{H}_{1}\right), \mathbf{z}_{q} \rightarrow \mathcal{H}_{1} .
$$

As formulated in [14], assuming that $\mathbf{z}_{q}$ presents a Gaussian distribution, the conditional PDF is given by

$$
\begin{aligned}
p\left(\mathbf{z}_{q} \mid \mathcal{H}_{j}\right)=\frac{1}{(2 \pi)^{\rho / 2}\left|\mathbf{K}_{\mathcal{H}_{j}}\right|^{1 / 2}} & e^{-\frac{1}{2}\left(\mathbf{z}_{q}-\mathbf{u}_{\mathcal{H}_{j}}\right)^{T} \mathbf{K}_{\mathcal{H}_{j}}^{-1}\left(\mathbf{z}_{q}-\mathbf{u}_{\mathcal{H}_{j}}\right)},
\end{aligned}
$$

where $\mathbf{u}_{\mathcal{H}_{j}}=\mathbb{E}\left\{\mathbf{z}_{q} \mid \mathcal{H}_{j}\right\},|\cdot|$ denotes the determinant operator, and $\mathbf{K}_{\mathcal{H}_{j}}$ is the autocovariance matrix, which can be expressed as

$$
\mathbf{K}_{\mathcal{H}_{j}}=\mathbb{E}\left\{\left(\mathbf{z}_{q}-\mathbf{u}_{\mathcal{H}_{j}}\right)\left(\mathbf{z}_{q}-\mathbf{u}_{\mathcal{H}_{j}}\right)^{\dagger}\right\}
$$

where $(\cdot)^{\dagger}$ is the Hermitian operator.

The second technique employed in this work to detect the status of the quanta is a MLP neural network [15]. This class of artificial neural network (ANN) was chosen due to its ability to separate regions with nonlinear borders, which can be applied without the knowledge of the statistics of the analyzed data. Therefore, the state-space formulation of the MLP with one hidden layer is given by

$$
\begin{gathered}
\mathbf{u}_{q}=\boldsymbol{\alpha}^{T}\left[\begin{array}{c}
\mathbf{z}_{q} \\
1
\end{array}\right], \\
y=\boldsymbol{\beta}^{T}\left[\begin{array}{c}
\tanh \left(\mathbf{u}_{q}\right) \\
1
\end{array}\right],
\end{gathered}
$$

in which $\mathbf{z}_{q}$ is the vector of features associated with the $q^{\text {th }}$ quantum, obtained from the procedure presented in (22); $\mathbf{u}_{q}=\left[\begin{array}{llll}u_{0} & u_{1} & \cdots & u_{L_{N-1}}\end{array}\right]^{T}$ is the domain of the activation function; $L_{N}$ is the number of neurons in the hidden layer of the network; $\boldsymbol{\alpha} \in \mathbb{R}^{\left(\mathbf{z}_{q}+1\right) \times L_{N}}$ is the matrix of synaptic weights between the network entrance and the hidden layer; and $\boldsymbol{\beta} \in \mathbb{R}^{\left(L_{N}+1\right) \times 1}$ is the matrix of synaptic weights between the hidden layer and the output of the MLP. The procedure proposed in [16] was used for training the MLP.

\section{Numerical Results}

The performance evaluation of the proposed spectral monitoring technique is based on the use of measured signals, which were obtained from a measurement campaign carried out in several homes in the city of Juiz de Fora, Brazil [17], when the frequency band between 1.7 and $100 \mathrm{MHz}$ is considered. Measurements of the noise sensed by an antenna and by a PLC coupler connected to the indoor and low-voltage electric power grid were obtained. The data acquisition system was constituted of an industrial computer, a Peripherical Component Interconnect (PCI) card with a 14-bit analog-todigital converter (ADC) at a rate of acquisition of 200 Mega samples per second, a PLC coupler, and an omnidirectional antenna. For the time-frequency mapping, $N=128$ and the following quanta dimensions were considered: $N_{T} \times N_{F} \in$ $\{1 \times 4,1 \times 8,4 \times 4,4 \times 8,8 \times 4,8 \times 8\}$.

Based on the measured data, we have noted that only the energy of each quantum is a useful feature for detection purposes since the other studied features, when compared to energy, did not present significant FDR values. Such information obtained from the measured data is rather relevant since it simplifies the design of a technique for detecting holes in the monitored spectrum. The numerical results discussed below are based on the use of $\mathbf{z}_{q}$ constituted by the energy values of the signals obtained from both air and power line.

Therefore, the spectral monitoring technique with its distinct configurations is evaluated in terms of detection rate $\left(P_{D}\right)$ of the unoccupied quanta, i.e., of holes in the spectrum, as well as false alarm rate $\left(P_{F}\right)$. In order to analyze the signals monitored in the time-frequency domain, time-frequency mapping is used, based on the DFT, DHT, MCLT, MTM, and HMTM transforms. Thus, monitoring technique configurations are formed by the combination of time-frequency transforms with detectors based on Bayes and MLP, presented in Section III-E, as shown in TABLE I.

TABLE I

CONFIGURATIONS OF THE PROPOSED SPECTRUM SENSING TECHNIQUE.

\begin{tabular}{lccccc} 
& DFT & DHT & MCLT & MTM & HMTM \\
\cline { 2 - 6 } Bayes & BDFT & BDHT & BMCLT & BMTM & BHMTM \\
MLP & MDFT & MDHT & MMCLT & MMTM & MHMTM \\
\hline
\end{tabular}

For each sensor element (antenna or PLC coupler), the data set with 2000 quanta was used, in which 1000 quanta were used in the training set and the other 1000 quanta were used to evaluate the performance in terms of the detection rate of holes in the spectrum and false alarm rate.

Fig. 3 and Fig. 4 show the performance, based on the detection rate $\left(P_{D}\right)$ and the false alarm rate $\left(P_{F}\right)$, of the monitoring technique using the captured signal with various quanta orders. First, it is notable that the Bayes-based configurations present worse performance values in both detection 
rate and false alarm rate when compared to the MLP-based ones. Also, regarding the detection rate for the considered configurations, it is remarkable the contrast of the results presented by the quanta $\Psi_{4 \times 8}^{a}$ and $\Psi_{8 \times 4}^{a}$, which correspond to the quanta obtained from the antenna. Moreover, it is noted that BDHT and BHMTM are the configurations most affected by the reduction in the size of the monitoring quantum. It is important to emphasize as well the high values of false alarm rate yielded by the quanta from the PLC coupler, especially when Bayes-based configurations are considered, with exception to BMCLT. Finally, note that MDHT and MHMTM configurations present higher values of false alarm rate when compared to the other MLP-based ones.

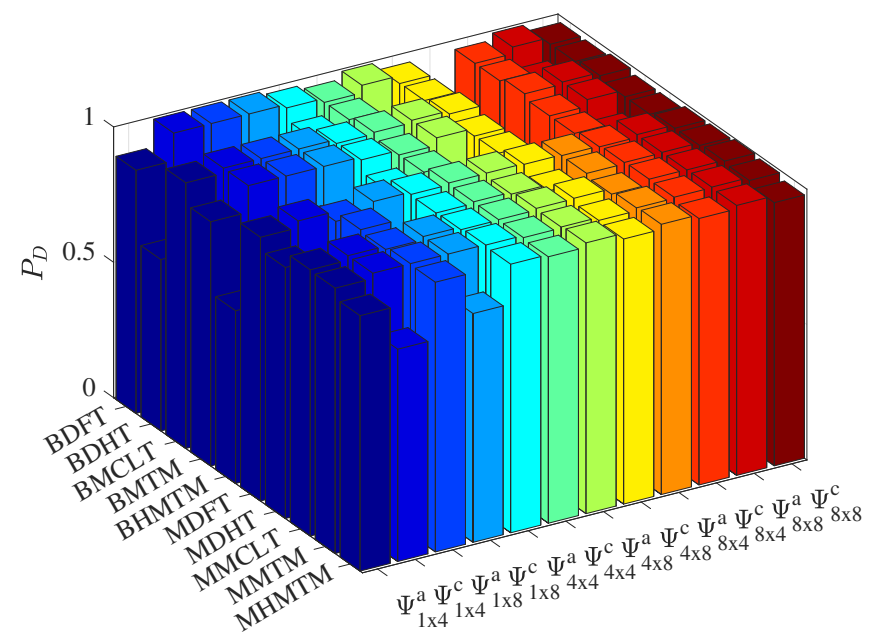

Fig. 3. Detection rate with several quanta.

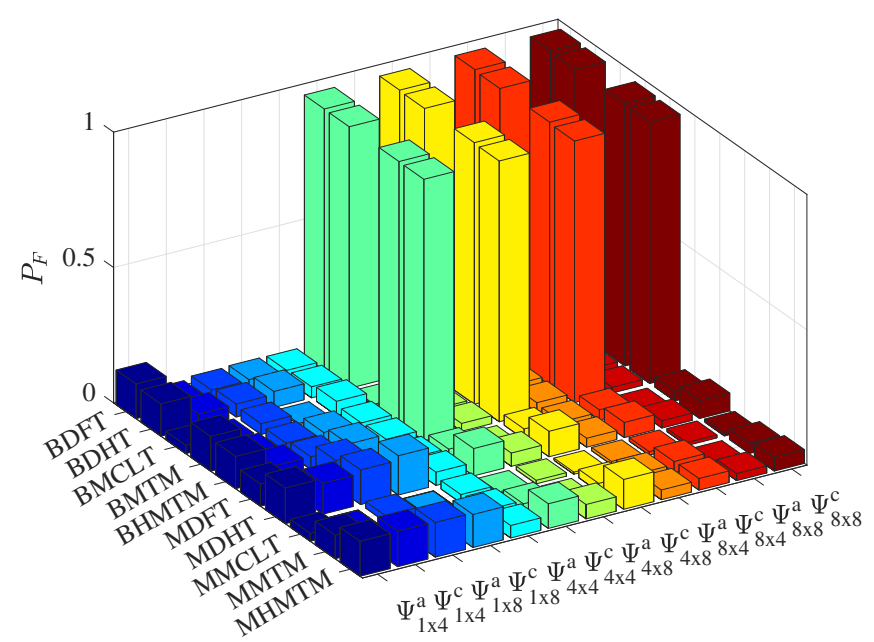

Fig. 4. False alarm rate with several quanta.

\section{CONClusion}

In the present work, an improved spectral monitoring sensing for cognitive PLC systems was proposed and formulated. In order to evaluate this proposal, a data set obtained from a measurement campaign containing noise measurements sensed by an antenna and a PLC coupler was adopted. Results showed that the performance of the MLP-based technique configurations are superior to the Bayes-based ones. This suggests that the statistical distribution of the monitoring quanta may not be modeled by a Gaussian distribution, which is an assumption made for Bayes detectors. Finally, the PLC coupler quanta presented considerably higher values of false alarm rate when compared to the antenna quanta.

\section{REFERENCES}

[1] K. Ali, A. X. Liu, I. Pefkianakis, and K.-H. Kim, "Distributed spectrum sharing for enterprise powerline communication networks," in IEEE International Conference on Network Protocols, Sep. 2018, pp. 367 377.

[2] J. A. Cortés and J. M. Idiago, "Smart metering systems based on power line communications," in Smart Grids and Their Communication Systems, E. Kabalci and Y. Kabalci, Eds. Springer Singapore, 2019, pp. 121-170.

[3] P. Pagani, R. Razafferson, A. Zeddam, B. Praho, M. Tlich, J.-Y. Baudais, A. Maiga, O. Isson, G. Mijic, K. Kriznar, and S. Drakul, "Electro magnetic compatibility for power line communications," in IEEE International Symposium on Personal, Indoor and Mobile Radio Communications, Sep. 2010, pp. 2799-2804.

[4] J. Adu Ansere, G. Han, H. Wang, C. Choi, and C. Wu, "A reliable energy efficient dynamic spectrum sensing for cognitive radio IoT networks," IEEE Internet of Things Journal, vol. 6, no. 4, pp. 6748-6759, Aug. 2019.

[5] A. Schwager, "Cognitive frequency exclusion in EN 50561-1:2012," in MIMO Power Line Communications: Narrow and Broadband Standards, EMC, and Advanced Processing, L. T. Berger, A. Schwager, P. Pagani, and D. Schneider, Eds. USA: CRC Press, 2014, ch. 22, pp. 601-624.

[6] B. Praho, M. Tlich, P. Pagani, A. Zeddam, and F. Nouvel, "Cognitive detection method of radio frequencies on power line networks," in IEEE International Symposium on Power Line Communications and Its Applications, Mar. 2010, pp. 225-230.

[7] Y. Lu, W. Liu, J. Li, and H. Gao, "Measurement and cognitive detection method of broadcast radio stations in distribution networks," in IEEE International Symposium on Power Line Communications and Its Applications, Mar. 2014, pp. 75-80.

[8] R. Vuohtoniemi, J. Vartiainen, J.-P. Mäkelä, and J. Iinatti, "Detection of wireless signals in broadband cognitive PLC using the modified 2-D LAD ACC algorithm," in IEEE International Symposium on Power Line Communications and Its Applications, Mar. 2015, pp. 234-238.

[9] L. R. Amado, E. S. C. Losqui, F. P. V. de Campos, A. A. M. de Medeiros, and M. V. Ribeiro, "Spectrum sensing for powering power line communications," in XXX Simpósio Brasileiro de Telecomunicações, Jan. 2012.

[10] M. S. Gupta and K. Kumar, "Progression on spectrum sensing for cognitive radio networks: A survey, classification, challenges and future research issues," Journal of Network and Computer Applications, vol. 143, pp. 47-76, Oct. 2019.

[11] J. Mendel, "Tutorial on higher-order statistics (spectra) in signal processing and system theory: theoretical results and some applications,' Proceedings of the IEEE, vol. 79, no. 3, pp. 278-305, Mar. 1991.

[12] D. D. Ferreira, A. S. Cerqueira, C. A. Duque, and M. V. Ribeiro, "HOS-based method for classification of power quality disturbances," Electronics Letters, vol. 45, pp. 183-185, Jan. 2009.

[13] T. R. Oliveira, P. Correia de Sá, S. L. de Paula Barbosa, M. V. Ribeiro, and C. A. G. Marques, "HOS-based impulsive noise detection technique for power line communication systems," in IEEE International Symposium on Power Line Communications and its Applications, Mar. 2010, pp. 125-130.

[14] M. V. Ribeiro, C. A. G. Marques, C. A. Duque, A. S. Cerqueira, and J. L. R. Pereira, "Detection of disturbances in voltage signals for power quality analysis using HOS," EURASIP Journal on Applied Signal Processing, vol. 1, pp. 177-177, Dec. 2007.

[15] S. Haykin, Neural networks and learning machines, 3rd ed. Upper Saddle River, NJ: Pearson Education, 2009.

[16] M. V. Ribeiro, J. G. A. Barbedo, J. M. T. Romano, and A. Lopes, "Fourier-lapped multilayer perceptron method for speech quality assessment," EURASIP Journal on Advances in Signal Processing, pp. 14251434, Jun. 2005.

[17] L. R. Amado, "Uma contribuição à análise de técnicas de monitoramento de espectro para sistemas PLC," Master's thesis, Federal University of Juiz de Fora, Juiz de Fora, Brazil, 2011. 